\title{
Required modifications and designated member reviews
}

Larry Covelli, the chairman of the Great Eastern University IACUC, was unwinding in his office when Sandy White, the school's Attending Veterinarian, walked in and sat herself down. "You know, Larry," she said, "I'm still totally confused about the OLAW notice on how to use designated member reviews (DMRs) after full committee reviews in order to clean up loose ends on a protocol ${ }^{1}$. I thought that the IACUC already had the authority to use DMRs, to ask questions and to require modifications to a protocol to secure its approval."

"Well, yes," responded Covelli, "but the notice you're talking about refers to questions that the committee has about a protocol when important information is missing, not specific modifications requested by the IACUC. A required modification would be something like changing the dose of an anesthetic to one required by the committee or requiring the use of an IACUC-defined earlier study endpoint. If the Principal Investigator (PI) agrees to accept the required modification and revises the protocol to include it, the protocol can be administratively accepted. A question, on the other hand, is a simple query, like asking for a stronger justification for the number of animals requested. It still requires full committee or designated member review of the response."

"I understand that," said White, "and that's why I'm confused. The first thing the notice says is that the IACUC may have questions because some significant information is lacking in the protocol. You know as well as I do that happens quite often. But then the notice refers to using the DMR process to approve modifications made to the protocol as a result of the answers to those questions. It seems to me that having a question for a $\mathrm{PI}$ and requiring modifications to secure approval are not one and the same. Do you see why I'm confused? There is already a process for handling each of those issues. If the IACUC has questions, it can do one of the two things we already do. We table the protocol, send the questions to the PI and then discuss the protocol again at the next meeting after the PI responds. Or we withhold approval, send our concerns to the PI, let him clean up the protocol and then re-review it like it was a new protocol, using the DMR process."

Covelli thought for a moment, opened his personal "rules and regulations" book and re-read the National Institutes of Health (NIH) notice. After a minute, he turned to White and said, "You know,
Sandy, we already have the authority to handle the situations you described, and the last part of the NIH notice actually says that we can continue to do what we've done in the past. My guess is that the whole idea of the notice is to make it easier to do a DMR without going through the process of notifying all the members and waiting to get their approval for using DMR. So maybe it's just a time-saver. But I do see your point. Getting an answer to a question is not the same as having a PI agree to an IACUC-required modification that's needed to secure approval. It's just confusing terminology, but I don't think we have to change the way we do business."

Is Covelli's explanation of what constitutes a required modification correct? Is White correct in claiming that OLAW notice NOT-OD-09-035 confuses questions with required modifications? Do you think that the notice simply expedites the protocol review process?

1. Office of Laboratory Animal Welfare. Guidance to IACUCs Regarding Use of Designated Member Review (DMR) for Animal Study Proposal Review Subsequent to Full Committee Review (FCR). Notice NOT-0D-09-035. (National Institutes of Health, Washington, DC; 8 January 2009). http://grants.nih.gov/grants/ guide/notice-files/NOT-0D-09-035.html

\section{RESPONSE}

\section{Expedite the process}

\section{F. Claire Hankenson, DVM, MS, DACLAM \& Troy Hallman, MS, VMD, DACLAM}

The casual conversation held between Covelli and White is probably not unusual for determining how to integrate $\mathrm{NIH}$ interpretations of Public Health Service (PHS) Policy requirements ${ }^{1}$ into existing institutional methods.
The new guidance under discussion (NOT-OD-09-035) $)^{2}$ was developed in response to questions from the 'research community' and enumerates instructions for animal care committees, regardless of membership numbers and institutional approaches to protocol reviews.

In general, the mechanisms for review, whether designated member review (DMR), full committee review (FCR) or a combination thereof, are dictated by individual institutional needs and program scope. The language in PHS
Policy ${ }^{1}$ section IV.C. 2 concerning FCR states that protocol approval may be granted 'only after review at a convened meeting of a quorum of the IACUC with the approval vote of a majority of the quorum present'. If a protocol reviewed by FCR is not approved owing to lack of information, the new guidance clarifies that every member of the IACUC must be present, or have otherwise signed a formalized proxy, to allow all opinions on a FCR to be heard or represented by designated committee members. 